\title{
Discovery of Four Doubly Imaged Quasar Lenses from the Sloan Digital Sky Survey
}

\author{
Naohisa Inada, ${ }^{1}$ Masamune Oguri, ${ }^{2,3}$ Cristian E. Rusu, ${ }^{4,5}$ Issha Kayo, ${ }^{6}$ and Tomoki \\ Morokuma $^{7}$
}

\begin{abstract}
We report the discovery of four doubly imaged quasar lenses. All the four systems are selected as lensed quasar candidates from the Sloan Digital Sky Survey data. We confirm their lensing hypothesis with additional imaging and spectroscopic follow-up observations. The discovered lenses are SDSS J0743+2457 with the source redshift $z_{s}=2.165$, the lens redshift $z_{l}=0.381$, and the image separation $\theta=1^{\prime \prime} \cdot 034$, SDSS J1128+2402 with $z_{s}=1.608$ and $\theta=$ $0^{\prime \prime} .844$, SDSS J1405+0959 with $z_{s}=1.810, z_{l} \approx 0.66$, and $\theta=1^{\prime \prime} .978$, and SDSS J1515+1511 with $z_{s}=2.054, z_{l}=0.742$, and $\theta=1^{\prime \prime} .989$. It is difficult to estimate the lens redshift of SDSS J1128+2402 from the current data. Two of the four systems (SDSS J1405+0959 and SDSS J1515+1511) are included in our final statistical lens sample to derive constraints on dark energy and the evolution of massive galaxies.
\end{abstract}

Subject headings: gravitational lensing: strong — quasars: general

\footnotetext{
${ }^{1}$ Department of Physics, Nara National College of Technology, Yamatokohriyama, Nara 639-1080, Japan.

${ }^{2}$ Department of Physics, Graduate School of Science, University of Tokyo, Hongo 7-3-1, Bunkyo-ku, Tokyo 113-0033, Japan.

${ }^{3}$ Kavli Institute for the Physics and Mathematics of the Universe, The University of Tokyo, 5-1-5 Kashiwano-ha, Kashiwa, Chiba 277-8568, Japan.

${ }^{4}$ Optical and Infrared Astronomy Division, National Astronomical Observatory of Japan, 2-21-1, Osawa, Mitaka, Tokyo 181-8588, Japan.

${ }^{5}$ Department of Astronomy, Graduate School of Science, University of Tokyo 7-3-1, Hongo Bunkyo-ku, Tokyo 113-0033, Japan.

${ }^{6}$ Department of Physics, Toho University, Funabashi, Chiba 274-8510, Japan.

${ }^{7}$ Institute of Astronomy, Faculty of Science, University of Tokyo, 2-21-1 Osawa, Mitaka, Tokyo 181-0015, Japan.
} 


\section{Introduction}

Gravitationally lensed quasars have proven to be a useful tool for a variety of astrophysical and cosmological studies, as described e.g., in Kochanek (2006). Applications of quasar lenses include constraints on cosmological parameters (e.g., Refsdal 1964; Turner 1990; Fukugita et al. 1990) and measurements of mass distributions for galaxy- and clusterscale halos (e.g., Kochanek 1991; Mao \& Schneider 1998; Williams \& Saha 2004; Oguri et al. 2013), interstellar medium associated with lensing or intervening galaxies (e.g., Curran et al. 2007; Cooke et al. 2010), and the structure of quasar accretion disk and outflow (e.g., Poindexter et al. 2008; Misawa et al. 2007). Many of these applications take advantage of the time-variable nature of quasars and therefore are not available for galaxy-galaxy strong lenses. The use of these quasar lenses can be enhanced by constructing a homogeneous lens sample with a well-characterized selection function. For instance, the Cosmic Lens All Sky Survey (CLASS; Myers et al. 2003; Browne et al. 2003) has provided a complete sample of 22 strong lenses selected among $\sim 16,000$ radio sources, which has been used for a number of cosmological and astrophysical studies (e.g., Chae et al. 2006).

We have completed the SDSS Quasar Lens Search (SQLS; Oguri et al. 2006, 2008, 2012; Inada et al. 2008, 2010, 2012), which is the current largest lensed quasar survey using optical data of the Sloan Digital Sky Survey (SDSS; York et al. 2000). As a result, we have discovered more than 40 lensed quasars including four lensed quasars presented in this paper. We have also rediscovered many previously known lensed quasars located in the SDSS footprint (Walsh et al. 1979; Wevmann et al. 1980; Surdej et al. 1987; Magain et al. 1988; Bade et al. 1997; Oscoz et al. 1997; Schechter et al. 1998; Mvers et al. 1999; Morgan et al. 2001; Winn et al. 2002; Reimers et al. 2002; Jackson et al. 2008, 2009, 2012). The total number of lensed quasars identified in the SDSS data is 621 (Inada et al. 2012). The statistical analysis for SQLS lenses to constrain both cosmological parameters and the evolution of massive galaxies has been reported in Oguri et al. (2012).

In this paper, we report the discovery of four lensed quasars from the SQLS. Since the description of the four lensed quasars in Inada et al. (2012) is so brief, we show the detailed properties of the four systems here. We briefly describe the SDSS data and our algorithm to locate lensed quasar candidates in $\S 2$. We then present follow-up observations to confirm their lensing hypotheses in $\S 3$. We carry out mass modeling in $\S$, Our result is summarized in $\S 5$.

\footnotetext{
${ }^{1}$ The gravitational lens nature of an object is not secure (Rusu et al. 2013).
} 


\section{Candidate Selection from the Sloan Digital Sky Survey}

The SQLS identifies lensed quasars among 100,000 quasars (Schneider et al. 2010) from the SDSS Data Release 7 (DR7). The SDSS is a survey using a dedicated widefield 2.5-meter telescope (Gunn et al. 2006) at the Apache Point Observatory (New Mexico, USA) to conduct imaging and spectroscopic surveys for a quarter of the sky. The imaging data were obtained through five broad-band filters (ugriz, Fukugita et al. 1996; Gunn et al. 1998; Doi et al. 2010) with astrometric accuracy better than $\sim 0$.'1 (Pier et al. 2003) and with photometric zeropoint accuracy better than $\sim 0.01 \mathrm{mag}(\operatorname{Hogg}$ et al. 2001; Smith et al. 2002; Ivezić et al. 2004; Tucker et al. 2006; Padmanabhan et al. 2008). Quasar and galaxy candidates were selected by the SDSS spectroscopic pipeline based on the imaging data (Eisenstein et al. 2001; Richards et al. 2002; Strauss et al. 2002). The candidates were tiled in each plate according to the algorithm of Blanton et al. (2003), and were observed with multi-object fiber spectrographs $(R \sim 1800)$ covering $3800 \AA$ to $9200 \AA$ (Blanton et al. 2003). The SDSS DR7 data have already been made public (Stoughton et al. 2002; Abazaijian et al. 2003, 2004, 2005, 2009; Adelman-McCarthy et al. 2006, 2007, 2008).

The procedure to identify lensed quasar candidates from the spectroscopic SDSS quasars has been described in the series of SQLS papers (Oguri et al. 2006; Inada et al. 2008, 2010, 2012), which we outline here. We use two selection methods. One is to find quasars with nearby objects whose colors are similar to the quasars. This method is tagged as the "color selection". The color selection does not always work well for the SDSS data because of the relatively poor spatial resolution of SDSS imaging data, whose seeing sizes are comparable to typical image separations of quasar lenses. Therefore, in order to find lenses which are not deblended into multiple components in the SDSS data, we also exploit the so-called "morphological selection", in which we select spectroscopic quasars with extended morphologies as lensed quasar candidates. SDSS J0743+2457 and SDSS J1128+2402 are selected by the morphological selection, whereas SDSS J1405+0959 and SDSS J1515+1511 are selected by both the color selection and the morphological selection. The SDSS properties of these four candidates are summarized in Table 1. We also show the SDSS $i$-band images of the four objects in Figure 1.

We note that SDSS J0743+2457 and SDSS J1405+0959 have been reported as quasar lenses ULAS 074352.6+245743 and ULAS 140515.4+095931, respectively, by the Major UKIDSS-SDSS Cosmic Lens Survey (MUSCLES) survey (Jackson et al. 2012). In the MUSCLES survey lens candidates are selected by cross-matching SDSS quasars with UKIDSS near-infrared images which typically have much better spatial resolution. Our identification of SDSS J0743+2457 and SDSS J1405+0959 as lens candidates is independent of the MUSCLES survey result. 


\section{Follow-up Imaging and Spectroscopy}

\subsection{Follow-up Data}

We carried out follow-up imaging and spectroscopic observations using the University of Hawaii 2.2-meter telescope (UH88), the Astrophysical Research Consortium 3.5-meter telescope (ARC), the 3.58-meter Telescopio Nazionale Galileo (TNG), the Subaru 8.2-m telescope, and the Gemini North 8.1-m telescope, to confirm the lensing hypothesis of the four lensed quasar candidates. All the four candidates are doubly imaged lens candidates, and therefore their lensing hypothesis should be confirmed by both similar spectral energy distributions (SEDs) for the two stellar components and the existence of an extended object between the two components that act as a lens. We summarize the details of our follow-up imaging and spectroscopic observations in Tables 2 and 3 .

We clearly detect two stellar components in each original image of the follow-up imaging observations. Once we subtract two Point Spread Functions (PSFs) using GALFIT (Peng et al. 2002), we detect a significant residual, indicating an additional component in between the two PSFs, for all four systems. We then model the system with two PSFs plus an extended component represented by a Sérsic profile. As shown in Figure 2, an extended component is clearly visible after subtracting the two PSFs. The Sérsic parameters of the best-fitting model are summarized in Table 4. We also summarize the astrometric and photometric results for each system in Table 5 .

Results of the spectroscopic follow-up observations are shown in Figure 3. For each candidate, we aligned the slit direction such that we can observe the two stellar components simultaneously. The good seeing condition (FWHM $\left.\lesssim 1^{\prime \prime} .0\right)$ enabled us to extract the spectrum of each component easily using standard IRAF2 tasks. The spectra indicate that the SEDs of the two stellar components are quite similar for all four systems. In particular, the two quasar components of the SDSS J1405+0959 system have very similar broad absorption, which is in strong support of strong lensing interpretation of this system. The fainter component of SDSS J1515+1511 shows a strong Mg II absorption at $\sim 5900 \AA$, which corresponds to an absorber at $z=0.742$, and is likely to be associated with the lensing galaxy (see below).

\footnotetext{
${ }^{2}$ IRAF is distributed by the National Optical Astronomy Observatories, which are operated by the Association of Universities for Research in Astronomy, Inc., under cooperative agreement with the National Science Foundation.
} 


\subsection{Lens Redshifts}

First we examine our follow-up spectra carefully to look for any spectral signature of lensing galaxies. We find that the spectrum of the fainter component of SDSS J0743+2457 contains a significant amount of galaxy flux. We extract the spectrum of the lensing galaxy $\mathrm{G}$ by subtracting the spectrum of image $\mathrm{A}$, with an appropriate offset corresponding to the flux ratio, from that of image B. Figure 4 clearly indicates that the lensing galaxy is an early-type galaxy at $z_{l}=0.381$.

For the other lens systems, we estimate redshifts of the lensing galaxies based on their colors. For SDSS J1405+0959, the Sérsic concentration index suggests that the residual may be a late-type galaxy. In this case, the $V-R$ and $R-I$ colors imply that the redshift is $z_{l} \sim 0.5$ (e.g., Fukugita et al. 1995). This is broadly consistent with $z_{l} \sim 0.66$ derived in Jackson et al. (2012) using the overall spectral shape of the lensing galaxy in their follow-up spectroscopic data. For SDSS J1515+1511, the morphology is more robust in the $i$-band, where it is consistent with a late-type galaxy. Its color $i-K^{\prime} \sim 4.4$ implies the redshift of $z \sim 0.8-1.1$, which is close to the redshift $z=0.742$ of the strong absorber seen in the spectrum of the fainter quasar image. Thus we tentatively assign its lens redshift to be $z_{l}=0.742$. It is difficult to estimate the photometric lens redshift of SDSS J1128+2402 from the current data.

\section{Mass Modeling}

We model the lens systems adopting a Singular Isothermal Ellipsoid (SIE) mass model using glafic (Oguri 2010). The number of parameters is eight (the position and flux of the source quasar, the position of the lensing galaxy, the Einstein radius, the ellipticity, and the position angle), while the number of constraints from the imaging observations is also eight (the positions and fluxes of the two quasar components, and the position of the lensing galaxy). Thus the model has zero degrees of freedom. We adopt the $I$-band imaging result as constraints for SDSS J0743+2457, SDSS J1128+2402, and SDSS J1405+0959. For SDSS J1515+1511, we use the $i$-band imaging result in spite of the lens being comparatively fainter, because the seeing was smaller, there were more stars available to build a PSF, and both astrometry and morphology are more robust. We summarize the parameters of the best-fitting $\left(\chi^{2} \sim 0\right)$ models in Table 6. As is common (e.g., Keeton et al. 1998), there are large differences in the lensing galaxy shapes between the observations (Table 4) and the best-fitting mass model parameters (Table 6). This may partly be explained by nearby perturbers which affect the lens potentials. 


\section{Summary}

We have confirmed the lensing hypothesis for four lens candidates selected by the SQLS, based on the similar SEDs of the stellar components together with the existence of extended objects in between the stellar components, and the successful mass modeling of the observed configurations. All four are doubly imaged quasar lens systems: SDSS J0743+2457 $\left(z_{s}=\right.$ 2.165, $z_{l}=0.381, \theta=1^{\prime \prime}$.034), SDSS J1128+2402 ( $z_{s}=1.608, \theta=0$ '.844), SDSS J1405+0959 $\left(z_{s}=1.810, z_{l} \sim 0.66, \theta=1^{\prime \prime} .978\right)$, and SDSS J1515+1511 $\left(z_{s}=2.054, z_{l}=0.742, \theta=\right.$ 1".989). Two of them (SDSS J1405+0959 and SDSS J1515+1511) are included in the SQLS statistical sample (Inada et al. 2012) and are used for cosmological constraints (Oguri et al. 2012).

These lens systems represent likely the last quasar lenses to emerge from the SQLS project. The applications of the SQLS lenses have been limited by the lack of high-resolution images for many of the SQLS lenses. We are therefore currently conducting a program to observe the SQLS lenses with Subaru Telescope laser guide start adaptive optics system to accurately measure the quasar image positions and light profile of the lensing galaxy (Rusu et al. 2011, C. E. Rusu et al., in preparation). We are also carrying out a lensed

quasar survey using the SDSS-III data (Eisenstein et al. 2011) from which we will be able to discover more quasar lenses.

This work was supported in part by the FIRST program "Subaru Measurements of Images and Redshifts (SuMIRe)", World Premier International Research Center Initiative (WPI Initiative), MEXT, Japan, and Grant-in-Aid for Scientific Research from the JSPS (23740161). I. K. acknowledges support from MEXT KAKENHI 24740171. Use of the UH 2.2-m telescope for the observations is supported by NAOJ. Based in part on observations obtained with the Apache Point Observatory 3.5-meter telescope, which is owned and operated by the Astrophysical Research Consortium, and on observations made with the Italian Telescopio Nazionale Galileo (TNG) operated on the island of La Palma by the Fundacion Galileo Galilei of the INAF (Istituto Nazionale di Astrofisica) at the Spanish Observatorio del Roque de los Muchachos of the Instituto de Astrofisica de Canarias. Based in part on observations obtained at the Gemini Observatory, which is operated by the Association of Universities for Research in Astronomy, Inc., under a cooperative agreement with the NSF on behalf of the Gemini partnership: the National Science Foundation (United States), the National Research Council (Canada), CONICYT (Chile), the Australian Research Council (Australia), Ministério da Ciência, Tecnologia e Inovação (Brazil) and Ministerio de Ciencia, Tecnología e Innovación Productiva (Argentina).

Funding for the SDSS and SDSS-II has been provided by the Alfred P. Sloan Foundation, 
the Participating Institutions, the National Science Foundation, the U.S. Department of Energy, the National Aeronautics and Space Administration, the Japanese Monbukagakusho, the Max Planck Society, and the Higher Education Funding Council for England. The SDSS Web Site is http://www.sdss.org/,

The SDSS is managed by the Astrophysical Research Consortium for the Participating Institutions. The Participating Institutions are the American Museum of Natural History, Astrophysical Institute Potsdam, University of Basel, Cambridge University, Case Western Reserve University, University of Chicago, Drexel University, Fermilab, the Institute for Advanced Study, the Japan Participation Group, Johns Hopkins University, the Joint Institute for Nuclear Astrophysics, the Kavli Institute for Particle Astrophysics and Cosmology, the Korean Scientist Group, the Chinese Academy of Sciences (LAMOST), Los Alamos National Laboratory, the Max-Planck-Institute for Astronomy (MPIA), the Max-Planck-Institute for Astrophysics (MPA), New Mexico State University, Ohio State University, University of Pittsburgh, University of Portsmouth, Princeton University, the United States Naval Observatory, and the University of Washington.

\section{REFERENCES}

Abazajian, K., et al. 2003, AJ, 126, 2081

Abazajian, K., et al. 2004, AJ, 128, 502

Abazajian, K., et al. 2005, AJ, 129, 1755

Abazajian, K. N., et al. 2009, ApJS, 182, 543

Adelman-McCarthy, J. K., et al. 2006, ApJS, 162, 38

Adelman-McCarthy, J. K., et al. 2007, ApJS, 172, 634

Adelman-McCarthy, J. K., et al. 2008, ApJS, 175, 297

Bade, N., Siebert, J., Lopez, S., Voges, W., \& Reimers, D. 1997, A\&A, 317, L13

Blanton, M. R., Lin, H., Lupton, R. H., Maley, F. M., Young, N., Zehavi, I., \& Loveday, J. 2003, AJ, 125, 2276

Browne, I. W. A., et al. 2003, MNRAS, 341, 13

Chae, K.-H., Mao, S., \& Kang, X. 2006, MNRAS, 373, 1369 
Cooke, R., Pettini, M., Steidel, C. C., King, L. J., Rudie, G. C., \& Rakic, O. 2010, MNRAS, 409, 679

Curran, S. J., Darling, J., Bolatto, A. D., Whiting, M. T., Bignell, C., \& Webb, J. K. 2007, MNRAS, 382, L11

Doi, M., et al. 2010, AJ, 139, 1628

Eisenstein, D. J., et al. 2001, AJ, 122, 2267

Eisenstein, D. J., et al. 2011, AJ, 142, 72

Fukugita, M., Ichikawa, T., Gunn, J. E., Doi, M., Shimasaku, K., \& Schneider, D. P. 1996, AJ, 111, 1748

Fukugita, M., Futamase, T., \& Kasai, M. 1990, MNRAS, 246, 24

Fukugita, M., Shimasaku, K., \& Ichikawa, T. 1995, PASP, 107, 945

Gunn, J. E., et al. 1998, AJ, 116, 3040

Gunn, J. E., et al. 2006, AJ, 131, 2332

Hogg, D. W., Finkbeiner, D. P., Schlegel, D. J., \& Gunn, J. E. 2001, AJ, 122, 2129

Inada, N., et al. 2008, AJ, 135, 496

Inada, N., et al. 2010, AJ, 140, 403

Inada, N., et al. 2012, AJ, 143, 119

Ivezić, Ž., et al. 2004, Astronomische Nachrichten, 325, 583

Jackson, N., Rampadarath, H., Ofek, E. O., Oguri, M., \& Shin, M.-S. 2012, MNRAS, 419, 2014

Jackson, N., Ofek, E. O., \& Oguri, M. 2009, MNRAS, 398, 1423

Jackson, N., Ofek, E. O., \& Oguri, M. 2008, MNRAS, 387, 741

Keeton, C. R., Kochanek, C. S., \& Falco, E. E. 1998, ApJ, 509, 561

Kobayashi, N., et al. 2000, SPIE, 4008, 1056 
Kochanek, C. S., Schneider, P., Wambsganss, J., 2006, Part 2 of Gravitational Lensing: Strong, Weak \& Micro, Proceedings of the 33rd Saas-Fee Advanced Course, G. Meylan, P. Jetzer \& P. North, eds. (Springer-Verlag: Berlin), 91

Kochanek, C. S. 1991, ApJ, 373, 354

Lawrence, A., et al. 2007, MNRAS, 379, 1599

Magain, P., Surdej, J., Swings, J.-P., Borgeest, U., \& Kayser, R. 1988, Nature, 334, 325

Mao, S., \& Schneider, P. 1998, MNRAS, 295, 587

Misawa, T., Inada, N., Ohsuga, K., Gandhi, P., Takahashi, R., \& Oguri, M. 2013, AJ, 145, 48

Miyazaki, S., et al. 2002, PASJ, 54, 833

Morgan, N. D., Becker, R. H., Gregg, M. D., Schechter, P. L., \& White, R. L. 2001, AJ, 121, 611

Myers, S. T., et al. 2003, MNRAS, 341, 1

Myers, S. T., et al. 1999, AJ, 117, 2565

Oguri, M. 2010, PASJ, 62, 1017

Oguri, M., et al. 2006, AJ, 132, 999

Oguri, M., et al. 2008, AJ, 135, 512

Oguri, M., et al. 2012, AJ, 143, 120

Oguri, M., et al. 2013, MNRAS, 429, 482

Oscoz, A., Serra-Ricart, M., Mediavilla, E., Buitrago, J., \& Goicoechea, L. J. 1997, AJ, 491, L7

Padmanabhan, N., et al. 2008, ApJ, 674, 1217

Peng, C. Y., Ho, L. C., Impey, C. D., \& Rix, H.-W. 2002, AJ, 124, 266

Pier, J. R., Munn, J. A., Hindsley, R. B., Hennessy, G. S., Kent, S. M., Lupton, R. H., \& Ivezić, Ź. 2003, AJ, 125, 1559

Poindexter, S., Morgan, N., \& Kochanek, C. S. 2008, ApJ, 673, 34 
Refsdal, S. 1964, MNRAS, 128, 307

Reimers, D., Hagen, H.-J., Baade, R., Lopez, S., \& Tytler, D. 2002, A\&A, 382, L26

Richards, G. T., et al. 2002, AJ, 123, 2945

Rusu, C. E., et al. 2011, ApJ, 738, 30

Rusu, C. E., et al. 2013, ApJ, 765, 139

Schechter, P. L., Gregg, M. D., Becker, R. H., Helfand, D. J., \& White, R. L. 1998, AJ, 115, 1371

Schneider, D. P., et al. 2010, AJ, 139, 2360

Smith, J. A., et al. 2002, AJ, 123, 2121

Stoughton, C., et al. 2002, AJ, 123, 485

Strauss, M. A., et al. 2002, AJ, 124, 1810

Surdej, J., Swings, J.-P., Magain, P., Courvoisier, T. J.-L., \& Borgeest, U. 1987, Nature, 329, 695

Tucker, D. L., et al. 2006, Astronomische Nachrichten, 327, 821

Turner, E. L. 1990, ApJ, 365, L43

Walsh, D., Carswell, R. F., \& Weymann, R. J. 1979, Nature, 279, 381

Weymann, R. J., et al. 1980, Nature, 285, 641

Williams, L. L. R. \& Saha, P. 2004, AJ, 128, 2631

Winn, J. N., Lovell, J. E. J., Chen, H.-W., Fletcher, A. B., Hewitt, J. N., Patnaik, A. R., \& Schechter, P. L. 2002, ApJ, 564, 143

York, D. G., et al. 2000, AJ, 120, 1579 
Table 1. SDSS Properties of the Lens Systems

\begin{tabular}{|c|c|c|c|c|c|c|c|c|}
\hline SDSS J0743+2457 & $07: 43: 52.61$ & $+24: 57: 43.6$ & $19.92 \pm 0.04$ & $19.42 \pm 0.02$ & $19.19 \pm 0.02$ & $19.09 \pm 0.02$ & $18.76 \pm 0.04$ & 2.165 \\
\hline SDSS J1128+2402 & $11: 28: 18.49$ & $+24: 02: 17.4$ & $18.35 \pm 0.02$ & $18.30 \pm 0.02$ & $18.12 \pm 0.02$ & $17.92 \pm 0.01$ & $17.92 \pm 0.02$ & 1.608 \\
\hline SDSS J1405+0959 & $14: 05: 15.40$ & $+09: 59: 29.3$ & $20.67 \pm 0.15$ & $20.52 \pm 0.13$ & $20.31 \pm 0.09$ & $19.72 \pm 0.09$ & $19.50 \pm 0.12$ & $\ldots$ \\
\hline \multirow[t]{2}{*}{ SDSS J1515+1511 } & $15: 15: 38.59$ & $+15: 11: 35.8$ & $18.43 \pm 0.04$ & $18.27 \pm 0.04$ & $18.23 \pm 0.04$ & $18.11 \pm 0.04$ & $17.91 \pm 0.04$ & 2.054 \\
\hline & $15: 15: 38.47$ & $+15: 11: 34.7$ & $18.94 \pm 0.09$ & $18.70 \pm 0.08$ & $18.61 \pm 0.07$ & $18.37 \pm 0.07$ & $18.19 \pm 0.07$ & $\cdots$ \\
\hline
\end{tabular}


Table 2. Summary of Imaging Follow-up Observations

\begin{tabular}{|c|c|c|c|}
\hline Object & Instruments & Observing Date (UT) & Exposure (s) \\
\hline SDSS J0743+2457 & $\operatorname{Tek} 2 \mathrm{k}(V R I z)$ & 2010Jan16(V), 2009Apr16(R), 2010Jan17(Iz) & $720(V), 300(R), 960(I z)$ \\
\hline SDSS J1128+2402 & $\operatorname{Tek} 2 \mathrm{k}(V I)$ & 2007Apr12(V), 2010Jan17(I) & $300(V), 960(I)$ \\
\hline SDSS J1515+1511 & $\operatorname{Tek} 2 \mathrm{k}(I), \operatorname{S}-\operatorname{cam}(i), \operatorname{IRCS}\left(K^{\prime}\right), \operatorname{SPIcam}(z)$ & 2009Apr16(I), 2011Mar31(i), 2011May7( $\left.K^{\prime}\right)$, 2011Mar26(z) & $400(I), 90(i), 1320\left(K^{\prime}\right), 1080(z)$ \\
\hline
\end{tabular}

Note. - The Tektronix $2048 \times 2048$ CCD camera (Tek2k) and the UH8k wide-field imager (UH8k) are installed on the UH88 telescope. The Suprime-Cam (S-cam; Mivazaki et al. 2002) and the Infrared Camera and Spectrograph (IRCS; Kobavashi et al. 2000) are installed on the Subaru telescope. The Seaver Prototype Imaging camera (SPIcam) is installed on the ARC telescope. 
Table 3. Summary of Spectroscopic Follow-up Observations

\begin{tabular}{ccccccc}
\hline \hline Object & Instrument & Slit width & Filter/Grism & Wavelength Coverage $(\AA)$ & Observing Date (UT) & Exposure (s) \\
\hline SDSS J0743+2457 & GMOS & $1^{\prime \prime}$ & GG455/R150 & $4600-9000$ & 2010 Mar 22 & 1200 \\
SDSS J1128+2402 & GMOS & $1^{\prime \prime}$ & GG455/R150 & $4600-9000$ & 2010 Mar 22 \\
SDSS J1405+0959 & DOLORES & $1^{\prime \prime}$ & LR-B & $3700-7800$ & 2008 Mar 1 & 900 \\
SDSS J1515+1511 & DOLORES & $1^{\prime \prime}$ & LR-B & $3700-7800$ & 2008 Apr 13 & 900 \\
\hline
\end{tabular}

Note. - The Gemini Multi-Object Spectrograph (GMOS) is installed on the Gemini telescope (North). The Device Optimized for the LOw RESolution (DOLORES) is installed on the TNG telescope. 
Table 4. Sérsic Parameters of the Lensing Galaxies

\begin{tabular}{ccccc}
\hline \hline Object & $r_{e}^{\mathrm{a}\left({ }^{\prime \prime}\right)}$ & $n^{\mathrm{b}}$ & $e^{\mathrm{c}}$ & $\theta_{e}\left(^{\circ}\right)^{\mathrm{c}}$ \\
\hline SDSS J0743+2457 & $0.58 \pm 0.24$ & $0.71 \pm 0.85$ & $0.47 \pm 0.16$ & $6.48 \pm 14.65$ \\
SDSS J1128+2402 & $0.33 \pm 0.06$ & $1.79 \pm 0.50$ & $0.49 \pm 0.03$ & $56.03 \pm 3.56$ \\
SDSS J1405+0959 & $0.37 \pm 0.05$ & $2.15 \pm 0.77$ & $0.06 \pm 0.11$ & $-17.02 \pm 81.33$ \\
SDSS J1515+1511 & $1.06 \pm 0.08$ & $1.08 \pm 0.20$ & $0.53 \pm 0.03$ & $-16.20 \pm 2.50$ \\
\hline
\end{tabular}

Note. - Best-fitting Sérsic parameters measured in the $I$-band images with GALFIT (The parameters for SDSS J1515+1511 are measured in the $i$-band. The Subaru S-cam $i$-band image has smaller seeing).

${ }^{\text {a} E f f e c t i v e ~ r a d i u s ~(i n ~ a r c s e c) ~ o f ~ t h e ~ S e ́ r s i c ~ p r o f i l e . ~}$

${ }^{\mathrm{b}}$ Sérsic concentration index.

${ }^{\mathrm{c}}$ Ellipticity and position angle measured East of North. 
Table 5. Relative Astrometry and Photometry of the Lens Systems

\begin{tabular}{|c|c|c|c|c|c|}
\hline Object & $\Delta X[\operatorname{arcsec}]$ & $\Delta Y[\operatorname{arcsec}]$ & $V$ & $R$ & $I$ \\
\hline \multicolumn{6}{|c|}{ SDSS J0743+2457 $\left(\theta=1{ }^{\prime \prime} 034 \pm 0.056\right)$} \\
\hline $\mathrm{A}$ & $\equiv 0.000$ & $\equiv 0.000$ & $19.33 \pm 0.01$ & $19.14 \pm 0.01$ & $18.90 \pm 0.02$ \\
\hline $\mathrm{B}$ & $-0.796 \pm 0.040$ & $-0.660 \pm 0.040$ & $21.03 \pm 0.06$ & $20.53 \pm 0.02$ & $20.36 \pm 0.31$ \\
\hline $\mathrm{G}$ & $-0.590 \pm 0.070$ & $-0.546 \pm 0.070$ & $\cdots$ & $\cdots$ & $20.47 \pm 0.37$ \\
\hline \multicolumn{6}{|c|}{ SDSS J1128+2402 $\left(\theta=00^{\prime \prime} 844 \pm 00^{\prime \prime} 006\right)$} \\
\hline $\mathrm{A}$ & $\equiv 0.000$ & $\equiv 0.000$ & $18.55 \pm 0.01$ & $\cdots$ & $18.48 \pm 0.02$ \\
\hline $\mathrm{B}$ & $-0.669 \pm 0.004$ & $0.515 \pm 0.004$ & $19.38 \pm 0.01$ & $\ldots$ & $19.15 \pm 0.03$ \\
\hline G & $-0.429 \pm 0.007$ & $0.361 \pm 0.007$ & $21.74 \pm 0.25$ & $\cdots$ & $18.56 \pm 0.06$ \\
\hline \multicolumn{6}{|c|}{ SDSS J1405+0959 $\left(\theta=1^{\prime \prime} \cdot 978 \pm 00^{\prime \prime} 013\right)$} \\
\hline $\mathrm{A}$ & $\equiv 0.000$ & $\equiv 0.000$ & $19.34 \pm 0.01$ & $19.18 \pm 0.01$ & $18.71 \pm 0.01$ \\
\hline $\mathrm{B}$ & $0.282 \pm 0.009$ & $-1.958 \pm 0.009$ & $20.43 \pm 0.01$ & $20.22 \pm 0.04$ & $19.90 \pm 0.05$ \\
\hline G1 & $0.021 \pm 0.021$ & $-1.614 \pm 0.021$ & $22.42 \pm 0.20$ & $20.59 \pm 0.04$ & $19.70 \pm 0.05$ \\
\hline G2 & $-1.553 \pm 0.024$ & $-0.792 \pm 0.024$ & $22.51 \pm 0.67$ & $21.25 \pm 0.09$ & $20.53 \pm 0.10$ \\
\hline \multicolumn{6}{|c|}{ SDSS J1515+1511 $\left(\theta=1^{\prime \prime} 989 \pm 00^{\prime \prime} 0.003\right)$} \\
\hline $\mathrm{A}$ & $\equiv 0.000$ & $\equiv 0.000$ & $i=18.15 \pm 0.01$ & $z=17.89 \pm 0.01$ & $K^{\prime}=15.80 \pm 0.01$ \\
\hline $\mathrm{B}$ & $1.648 \pm 0.002$ & $-1.114 \pm 0.002$ & $i=18.54 \pm 0.01$ & $z=18.32 \pm 0.02$ & $K^{\prime}=16.06 \pm 0.01$ \\
\hline G & $1.384 \pm 0.030$ & $-0.946 \pm 0.036$ & $i=21.39 \pm 0.06$ & $z=20.73 \pm 0.51$ & $K^{\prime}=17.03 \pm 0.04$ \\
\hline
\end{tabular}

Note. - Magnitudes are in the Vega system, except for $i$ - and $z$-band which are in AB system. The $K^{\prime}$ band photometric zero point for SDSS J1515+1511 is less robust, as it is based on a single star in the field of view (non-photometric conditions), with catalog uncertainty 0.11 mag. 
Table 6. Results of Mass Modeling

\begin{tabular}{ccccc}
\hline \hline Object & $R_{\mathrm{E}}\left({ }^{\prime \prime}\right)^{\mathrm{a}}$ & $e^{\mathrm{b}}$ & $\theta_{e}\left(^{\circ}\right)^{\mathrm{b}}$ & $\mu_{\mathrm{tot}}^{\mathrm{c}}$ \\
\hline SDSS J0743+2457 & 0.53 & 0.19 & 89.4 & 3.6 \\
SDSS J1128+2402 & 0.42 & 0.06 & -75.4 & 6.0 \\
SDSS J1405+0959 & 1.03 & 0.49 & 67.4 & 2.6 \\
SDSS J1515+1511 & 0.90 & 0.60 & -25.0 & 2.8 \\
\hline
\end{tabular}

${ }^{a}$ Einstein radius in arcsec.

${ }^{b}$ Ellipticity and position angle measured East of North.

'Total magnification factor predicted by the best fitting model. 

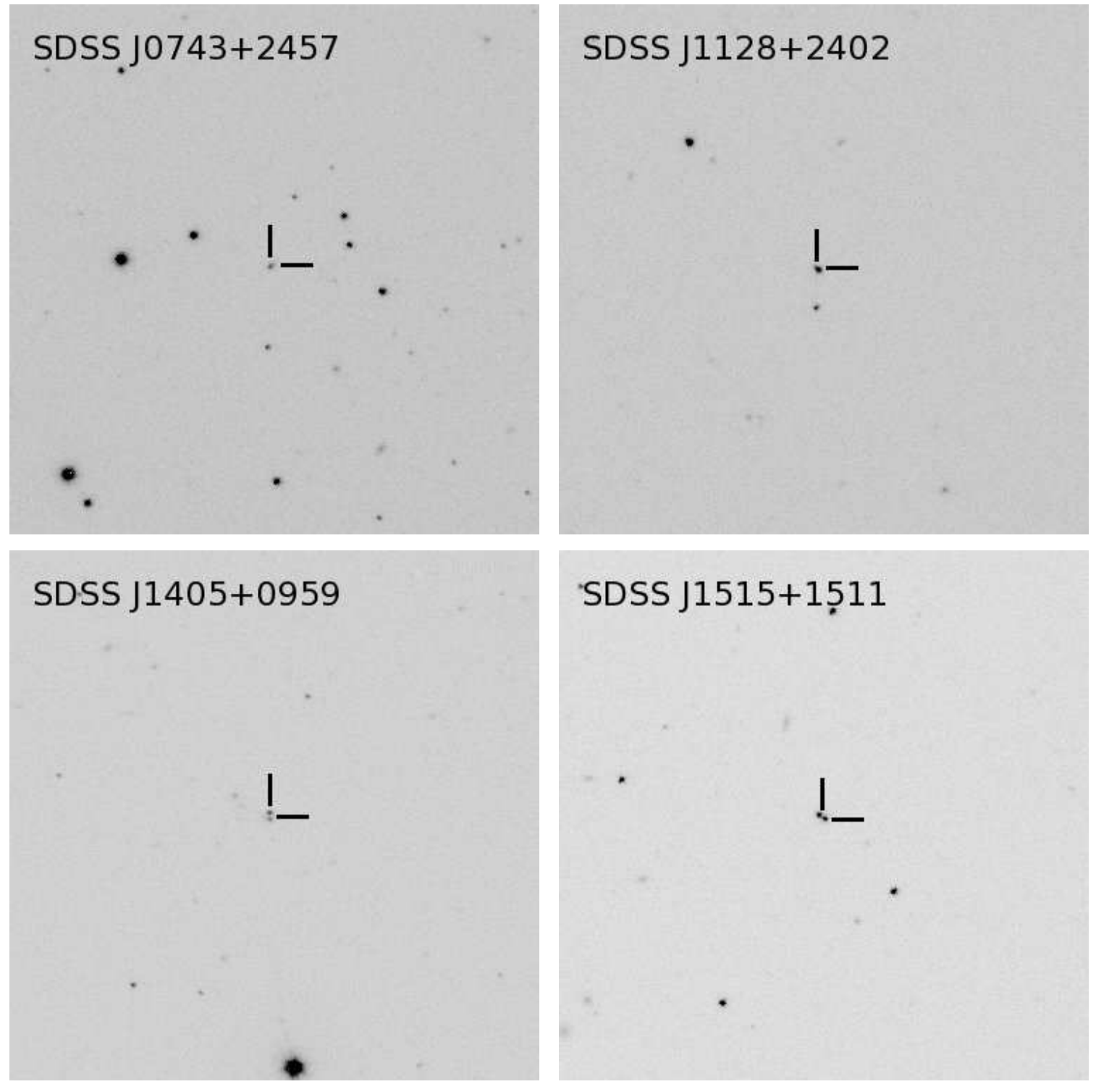

Fig. 1.- Finding charts (SDSS $i$-band images) of the 4 lens systems. The size of each figure is $2.5 \times 2.5$. North is up and East is left. 

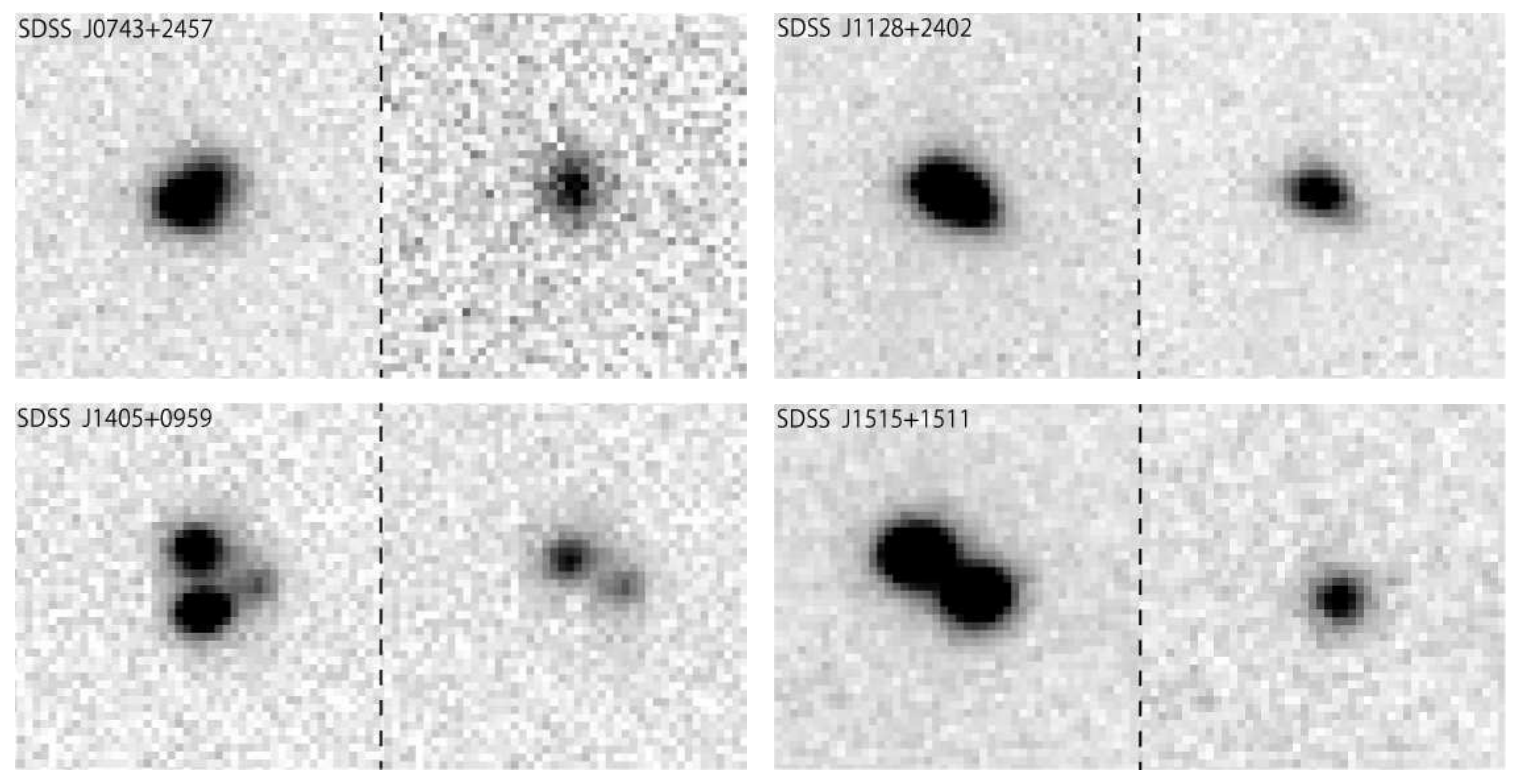

Fig. 2.- Follow-up I-band images of the 4 lens systems. The images of SDSS J0743+2457, SDSS J1128+2402, and SDSS J1515+1511 were taken with UH88/Tek2k (0'.22 pixel $\left.{ }^{-1}\right)$, and the image of SDSS J1405+0959 were taken with UH88/8k $\left(0^{\prime \prime} 235\right.$ pixel $\left.^{-1}\right)$. North is up and East is left. For each system, the left panel shows the original image and the right panel shows the image after subtracting two PSF components. The residuals in the right panels are all fitted well by the Sérsic profile. See Table 4 for the fitting results. 

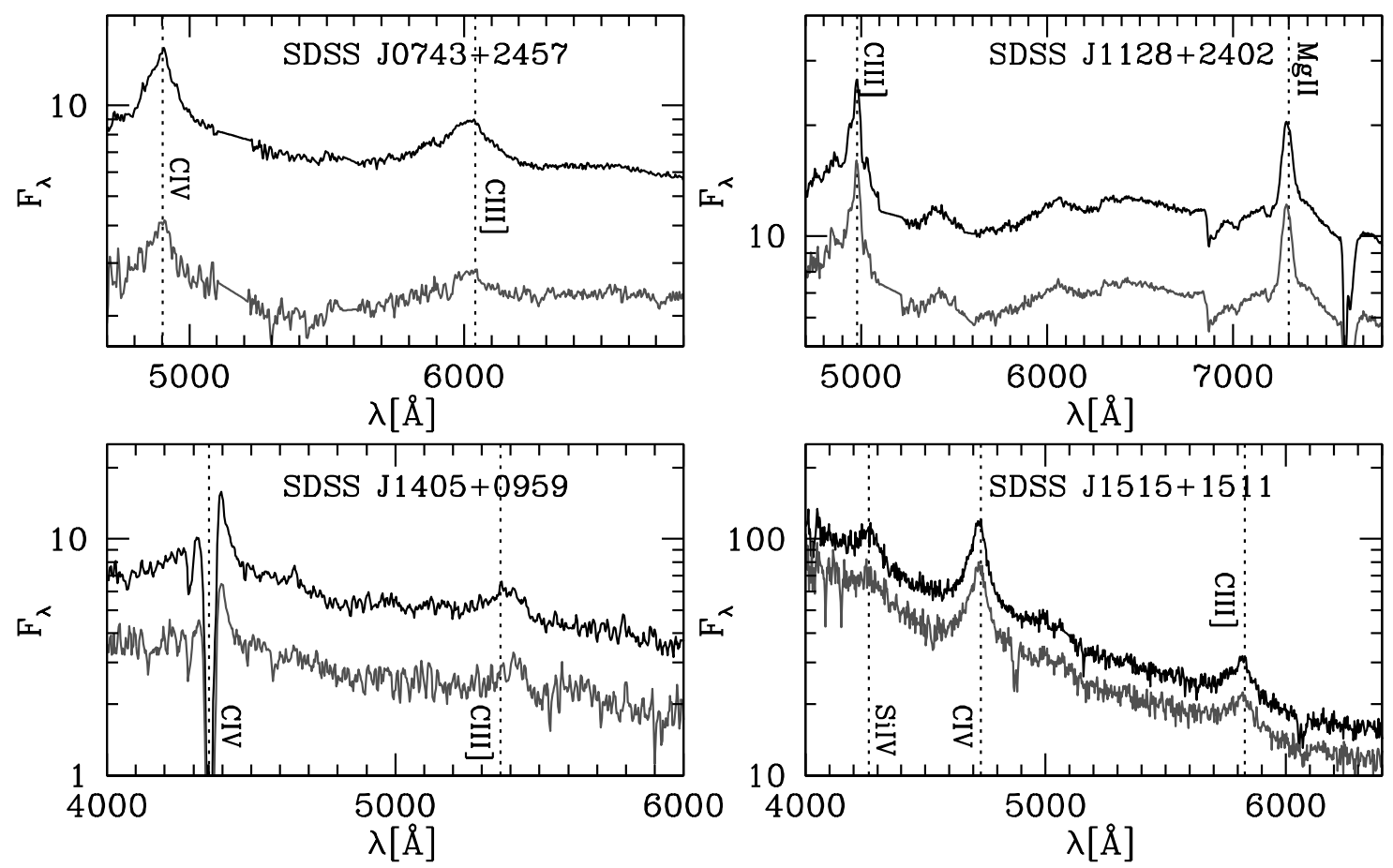

Fig. 3.- Spectra of two lensed quasar images. The data between $5100 \AA$ and $5200 \AA$ for SDSS J0743+2457 and SDSS J1128+2402 are excluded due to a gap of the detector. The wavelengths of quasar emission lines are indicated by vertical dotted lines. See Table 3 for details of our spectroscopic follow-up observations. 


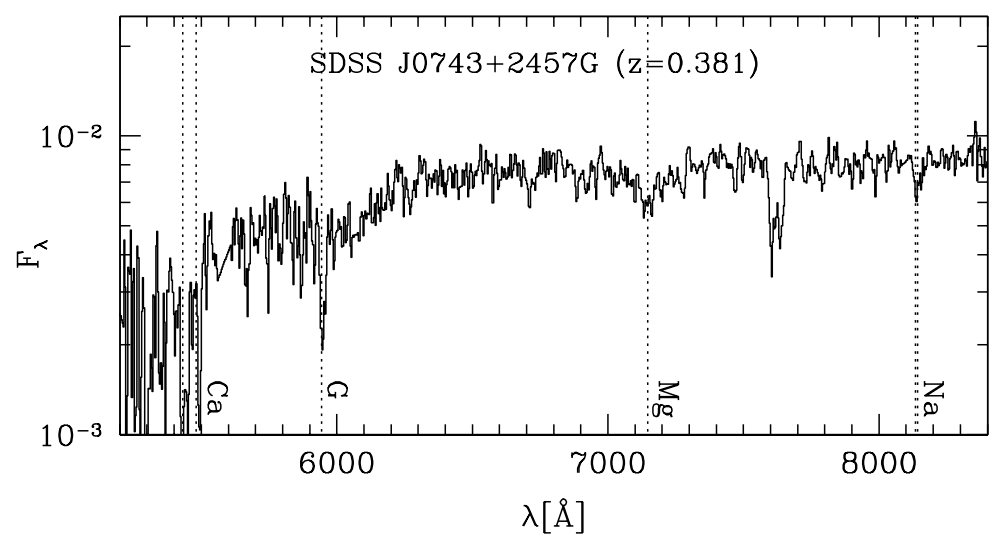

Fig. 4. - The spectrum of the lensing galaxy G of SDSS J0743+2457. The spectrum clearly indicates that the lensing galaxy is an early-type galaxy at $z=0.381$. 